\title{
Structure of the catalytic domain of a state transition kinase homolog from Micromonas algae
}

\author{
Jiangtao Guo ${ }^{1,2}$, Xuepeng Wei ${ }^{1,2}$, Mei Li ${ }^{1}$, Xiaowei Pan ${ }^{1}$, Wenrui Chang ${ }^{1 \bowtie}$, Zhenfeng Liu ${ }^{1 \bowtie}$ \\ ${ }^{1}$ National Laboratory of Biomacromolecules, Institute of Biophysics, Chinese Academy of Sciences, Beijing 100101, China \\ ${ }^{2}$ University of Chinese Academy of Sciences, Beijing 100039, China \\ $\triangle$ Correspondence: liuzf@sun5.ibp.ac.cn (Z. Liu), wrchang@sun5.ibp.ac.cn (W. Chang) \\ Received April 28, 2013 Accepted May 27, 2013
}

\begin{abstract}
Under natural environments, plants and algae have evolved various photosynthetic acclimation mechanisms in response to the constantly changing light conditions. The state transition and long-term response processes in photosynthetic acclimation involve remodeling and composition alteration of thylakoid membrane. A chloroplast protein kinase named Stt7/STN7 has been found to have pivotal roles in both state transition and longterm response. Here we report the crystal structures of the kinase domain of a putative Stt7/STN7 homolog from Micromonas sp. RCC299 (MsStt7d) in the apo form and in complex with various nucleotide substrates. MsStt7d adopts a canonical protein kinase fold and contains all the essential residues at the active site. A novel hairpin motif, found to be a conserved feature of the Stt7/STN7 family and indispensable for the kinase stability, interacts with the activation loop and fixes it in an active conformation. We have also demonstrated that MsStt7d is a dualspecificity kinase that phosphorylates both Thr and Tyr residues. Moreover, preliminary in vitro data suggest that it might be capable of phosphorylating a consensus $\mathrm{N}$-terminal pentapeptide of light-harvesting proteins $\mathbf{M i}$ cromonas Lhcp4 and Arabidopsis Lhcb1 directly. The potential peptide/protein substrate binding site is predicted based on the location of a pseudo-substrate contributed by the adjacent molecule within the crystallographic dimer. The structural and biochemical data presented here provide a framework for an improved understanding on the role of Stt7/STN7 in photosynthetic acclimation.
\end{abstract}

KEYWORDS Stt7/STN7 kinase, state transition, phosphorylation

\section{INTRODUCTION}

In order to adapt to constantly changing light conditions under natural environments, photosynthetic organisms have developed the ability to regulate distribution of excitation energy between photosystem II (PSII) and photosystem I (PSI), through a process known as state transition (Bonaventura and Myers, 1969; Murata, 1969). The efficiency of photosynthesis under fluctuating light conditions is thereby maintained at optimal level. During state transition, the major light-harvesting complex (LHCII) is reversibly phosphorylated and migrates between PSII and PSI to balance the excitation level of two photosystems (Bennett, 1991; Allen, 1992). The activity of the kinase responsible for LHCII phosphorylation was found to be regulated by the redox state of plastoquinone (PQ) pool (Allen et al., 1981) and cytochrome $b_{6} f$ (cyt $b_{6} f$ ) (Vener et al., 1997; Zito et al., 1999). Based on the present model of state transition (Wollman, 2001; Rochaix, 2007; Kargul and Barber, 2008), when the PSII is over-excited, the PQ pool is highly reduced and plastoquinol $\mathrm{PQH}_{2}$ binds to the $\mathrm{Q}_{0}$ site of cyt $b_{6} f$, leading to activation of LHCIl kinase which then phosphorylates LHCII. The phospho-LHCII migrates from PSII to PSI and redistributes energy to PSI (state II). Over-excitation of PSI leads to oxidation of the $\mathrm{PQ}$ pool and deactivation of LHCII kinase. The LHCIl phosphatase dephosphorylates phospho-LHCII, followed by migration of LHCII from PSI back to PSII. Thus, the energy absorbed by LHCII is delivered back to PSII (state I).

Searching for the LHCIl kinase is a central and longstanding goal in the field of state transition research (Lin et al., 1982; Coughlan and Hind, 1987; Snyders and Kohorn, 1999, 2001; Allen and Race, 2002). Recently, Stt7 from Chlamydomonas reinhardtii (CrStt7) and its ortholog STN7 from Arabidopsis thaliana (AtSTN7) have been reported as protein kinases located on the thylakoid membrane with crucial roles 
Table 1. Multiple Stt7/STN7 homologs from Micromonas sp. RCC299a

\begin{tabular}{lllllll}
\hline Accession & Name & Max score & Total score & Query coverage & E-value & Max identity \\
\hline XP_002499717 & MsStt7a & 291 & 291 & $98 \%$ & $2 \times 10^{-93}$ & $51 \%$ \\
XP_002504036 & MsStt7b & 231 & 231 & $94 \%$ & $3 \times 10^{-69}$ & $39 \%$ \\
XP_002500340 & MsStt7c & 221 & 221 & $98 \%$ & $1 \times 10^{-67}$ & $40 \%$ \\
XP_002504185 & MsStt7d & 149 & 149 & $98 \%$ & $8 \times 10^{-41}$ & $33 \%$ \\
XP_002505498 & MsStt7e & 145 & 145 & $97 \%$ & $4 \times 10^{-40}$ & $32 \%$ \\
\hline
\end{tabular}

${ }^{a}$ The top five results obtained by searching the Non-redundant protein sequence (nr) database of Micromonas sp. RCC299 in NCBI with the BLAST program using the kinase domain of AtSTN7 (Phe 134-Phe 452) as the query sequence (Altschul et al., 1997).

in state transition and phosphorylation of LHCll (Depège et al., 2003; Bellafiore et al., 2005). In the Stt7/STN7 deletion mutants, the state transition from state I to II is blocked and the phosphorylation of LHCll stays at low levels under changing light conditions (Depège et al., 2003; Bellafiore et al., 2005). The Stt7/STN7 protein contains a catalytic domain resembling Ser/Thr kinases, a predicted transmembrane helix, and a lumen-exposed $\mathrm{N}$-terminal region with a pair of conserved Cys residues which are important for the regulation of Stt7 activity (Depège et al., 2003; Lemeille et al., 2009). Although co-immunoprecipitation data showed that Stt7 may interact with LHCII (Lemeille et al., 2009), so far it remains unclear whether Stt7/ STN7 phosphorylates LHCII directly or indirectly by activating an unidentified LHCII kinase through an unknown signaling cascade. The structural basis for the phosphorylation of LHCII, as well as the regulatory mechanism of LHCIl kinase activity, is also largely unknown.

In addition to its role in the state transition (acting on a timescale of seconds to minutes), STN7 might also be involved in the long-term response (LTR, on a timescale of hours, days and seasons) of plants toward changes in light quality which leads to modification of thylakoid composition (Bonardi et al., 2005; Wagner et al., 2008; Pesaresi et al., 2009). It was proposed that STN7 phosphorylated an unidentified thylakoid protein that triggered LTR signaling processes (Pesaresi et al., 2009). Nevertheless, the detailed mechanism of LTR and the molecular basis for the function of STN7 in LTR await further investigation.

An in-depth understanding of the mechanistic roles of Stt7/ STN7 in state transition and LTR has been impeded by the lack of three-dimensional structure of Stt7/STN7. Here we report for the first time the crystal structures of the kinase domain of a putative Stt7/STN7 homolog from Micromonas sp. RCC299 (MsStt7d) in the apo form and in complex with nucleotide substrates. The phosphorylation behavior of MsStt7d, as well as the peptide substrate specificity and its potential binding site on MsStt7d, has been investigated in this work.

\section{RESULTS}

The kinase domain of MsStt7d adopts the canonical protein kinase fold

In an effort to characterize the structure and function of Stt7/
STN7 proteins, numerous homologs of Stt7/STN7 including those from Arabidopsis thaliana, Oryza sativa, Chlamydomonas reinhardtii and Micromonas sp. RCC299 have been cloned and overexpressed in $\mathrm{N}$-terminal truncation form covering the entire kinase domain. Among more than 50 constructs made, MsStt7d exhibited the best behavior with high expression level in soluble and stable form, while other homologs either expressed as inclusion body, or precipitated readily during purification. As one of the multiple putative Stt7/STN7 homologs found in Micromonas sp. RCC299 (Table 1), the MsStt7d contains a kinase domain (MsStt7d-KD, Arg 172-Phe 488) sharing $47 \%$ sequence similarity with the corresponding region of CrStt7 or AtSTN7 (Fig. 1). To reveal the molecular architecture of Stt7/STN7 kinase domain and to gain insights into its catalytic and regulatory mechanisms, we have determined the high-resolution crystal structures of MsStt7d-KD in the apo form, and in complex with ADP, ATP or AMP-PNP. Data collection and refinement statistics are listed in Table 2.

In the crystallographic asymmetric unit, there are two MsStt7d-KD molecules related by pseudo- $C_{2}$ symmetry (Fig. 2A). Each monomer contains the kinase domain of MsStt7d (Arg 172-Phe 488), and 21 additional amino acid residues (Leu 151-lle 171) at the N-terminal region (Fig. 2B). The kinase domain adopts a canonical protein kinase fold which can be divided into two lobes. The small $\mathrm{N}$-terminal lobe consists of a sheet of six antiparallel $\beta$-strands $(\beta 1-\beta 6)$ and a single $\alpha$-helix ( $\alpha 1$ ), while the large $C$-terminal lobe contains nine $\alpha$ helices $(\alpha 2-\alpha 10)$ and a pair of short $\beta$-strands ( $\beta 7$ and $\beta 8$ ) (Fig. 2B). The nucleotide substrate binding site is located in the cleft sandwiched between the two lobes. Within the N-lobe region, residues $178-186$ linking $\beta 2$ and $\beta 3$ form a highly conserved glycine-rich loop (with LGXGXXGXV motif, also known as the $\mathrm{P}$-loop) (Fig. $2 \mathrm{~B}$ ) that is usually involved in the binding of ATP, substrate recognition, catalysis and kinase activity regulation (Bossemeyer, 1994; Hanks and Hunter, 1995). In the $\mathrm{C}$-lobe region, there is another functionally important loop between residue 338-370 (Fig. 2B) corresponding to the activation loop in protein kinases. The activation loop may adopt variable conformations under inactive and active states, exerting an important role in the control of kinase activity (Nolen et al., 2004). 


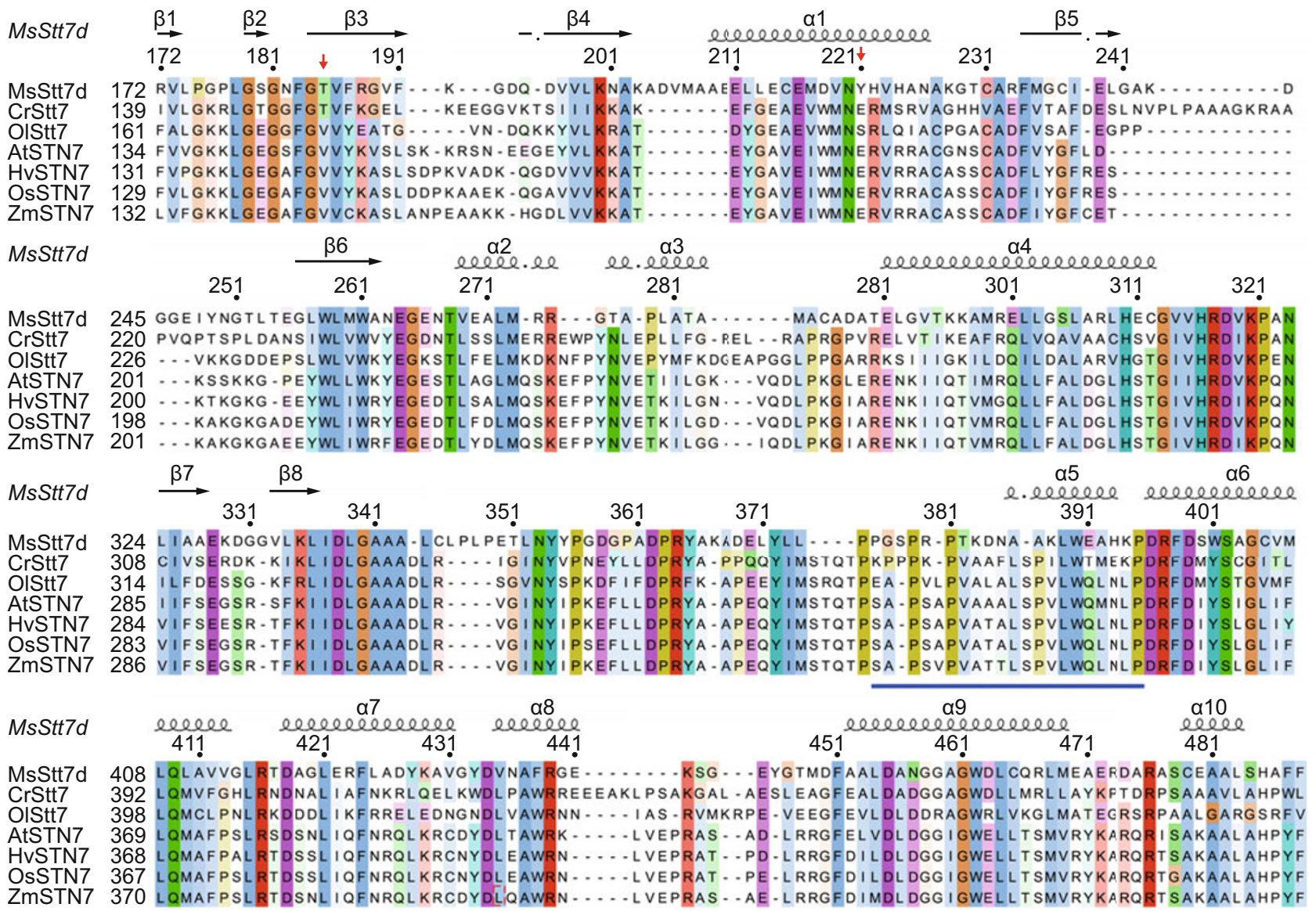

Figure 1. Sequence alignment of the protein kinase domains of various Stt7/STN7 family members. Multiple sequence alignment is performed with ClustalW2 followed by manual adjustment and displayed with Jalview using the coloring mode by conservation. The autophosphorylation sites on MsStt7d-KD are indicated with red arrows; the novel a5 hairpin region is highlighted by thick blue underline. Abbreviations: MsStt7d, a Stt7 homolog from Micromonas sp. RCC299; CrStt7, Stt7 from Chlamydomonas reinhardtii; OIStt7, Stt7 from Ostreococcus lucimarinus; AtSTN7, STN7 from Arabidopsis thaliana; HvSTN7, STN7 from Hordeum vulgare; OsSTN7, STN7 from Oryza sativa; ZmSTN7, STN7 from Zea mays.

As shown in Fig. 2C, MsStt7d-KD contains all the conserved residues essential for the binding of ATP and catalysis of phosphoryl-transfer reaction at the active site located within the inter-lobe cleft. Previous work on the cyclin-dependent protein kinase 2 (CDK2, a Ser/Thr kinase) has demonstrated that residues Lys 33, Asn 132 and Asp 145 are involved in the binding of ATP phosphate groups directly or through $\mathrm{Mg}^{2+}$ ion (De Bondt et al., 1993). The corresponding conserved residues in MsStt7d-KD are residues Lys 200, Asn 323 and Asp 338 according to the superposition of MsStt7d-KD structure with that of CDK2 (Fig. 2C). The role of these residues in binding nucleotide molecule is further confirmed in the structure of MsStt7dKD-ADP complex. In this structure, one ADP and one $\mathrm{Mg}^{2+}$ ion per monomer are found inside the active site (Fig. 2D). The $\mathrm{Mg}^{2+}$ ion is coordinated by the carbonyl of Asn 323 side chain, the carboxyl of Asp 338, two oxygen atoms from $\alpha$ - and $\beta$-phosphate groups, respectively, and two water molecules (Fig. 2D). The a-phosphate group of ADP molecule forms strong ionic interaction with the side chain amine group of Lys 200. A nearby conserved acidic residue, Glu 216 from the $\alpha 1$ helix stabilizes the orientation of Lys 200 side chain by forming ionic interaction with it (Fig. 2D). The Asp 318 in MsStt7d$\mathrm{KD}$ is deduced to be the catalytic residue with a similar role as the Asp 127 in CDK2, which coordinates the hydroxyl of the substrate for accepting the $\mathrm{y}$-phosphate from ATP (Fig. 2C) (De Bondt et al., 1993).

The structures of MsStt7d-KD in complex with ATP and AMP-PNP are nearly identical to the one in complex with ADP. In the MsStt7d-KD-ATP complex, the y-phosphate group of ATP could not be detected in the electron density map, presumably due to hydrolysis of ATP by the kinase during the process of crystal soaking (Fig. 2E). Previously, the intrinsic ATPase activity was also observed in other protein kinases and interpreted as the activity of catalyzing phosphoryl-transfer reaction toward water molecules (Ward and O'Brian, 1992; Mendelow et al., 1993). As for the MsStt7d-KD-AMP-PNP complex, 
Table 2. Data collection and refinement statistics

\begin{tabular}{|c|c|c|c|c|c|}
\hline Parameters & MsStt7d-KD-KI & Native MsStt7d-KD & MsStt7d-KD-ADP & MsStt7d-KD-ATP & MsStt7d-KD-AMP-PNP \\
\hline \multicolumn{6}{|l|}{ Data collection } \\
\hline Wavelength $(\AA)$ & 1.5418 & 0.97939 & 0.97853 & 0.97853 & 0.97853 \\
\hline Space group & $\mathrm{P} 2_{1}$ & $\mathrm{P} 2_{1}$ & $\mathrm{P} 2_{1}$ & $\mathrm{P} 2_{1}$ & $\mathrm{P} 2_{1}$ \\
\hline$a, b, c(\AA)$ & $\begin{array}{l}54.10,133.31 \\
55.30\end{array}$ & $\begin{array}{l}53.71,133.14 \\
55.05\end{array}$ & $\begin{array}{l}53.93,133.03 \\
55.16\end{array}$ & $\begin{array}{l}53.67,132.64 \\
55.01\end{array}$ & $\begin{array}{l}53.86,132.61 \\
55.18\end{array}$ \\
\hline$\beta\left(^{\circ}\right)$ & 116.65 & 116.30 & 116.49 & 116.26 & 116.64 \\
\hline Resolution $(\AA)$ & $33.3-2.26$ & $50-1.35$ & $50-1.50$ & $33.16-1.60$ & $33.15-1.70$ \\
\hline Unique reflections & 32604 & 150593 & 110828 & 90626 & 75812 \\
\hline Completeness (\%) & $99.4(97.7)^{\mathrm{a}}$ & $99.9(100.0)$ & $99.9(100.0)$ & $100.0(100.0)$ & $100.0(100.0)$ \\
\hline Redundancy & $12.9(12.4)$ & $4.0(3.7)$ & $7.5(7.4)$ & $6.9(7.1)$ & $7.2(7.2)$ \\
\hline$I / \sigma(I)$ & $20.9(7.7)$ & $29.0(2.8)$ & $32.9(4.5)$ & $14.4(3.5)$ & $14.8(4.1)$ \\
\hline$R_{\text {sym }}{ }^{\mathrm{b}}$ & $0.094(0.315)$ & $0.043(0.475)$ & $0.063(0.542)$ & $0.080(0.518)$ & $0.081(0.474)$ \\
\hline \multicolumn{6}{|l|}{ Refinement } \\
\hline Resolution $(\AA)$ & - & $50-1.35$ & $50-1.50$ & $33.16-1.60$ & $33.15-1.70$ \\
\hline$R_{\text {work }} / R_{\text {free }}{ }^{\mathrm{c}}$ & - & $0.133 / 0.174$ & $0.157 / 0.199$ & $0.168 / 0.193$ & $0.172 / 0.201$ \\
\hline Protein atoms & - & 5295 & 5281 & 5293 & 5280 \\
\hline Ligand/ion atoms & - & 4 & 57 & 56 & 65 \\
\hline Water atoms & - & 876 & 654 & 572 & 625 \\
\hline \multicolumn{6}{|l|}{ B-factors $\left(\AA^{2}\right)$} \\
\hline all & - & 20.3 & 23.5 & 21.6 & 26.6 \\
\hline protein & - & 18.8 & 22.4 & 21.0 & 25.9 \\
\hline ligand/ion & - & 17.4 & 23.4 & 36.1 & 36.5 \\
\hline water & - & 29.5 & 32.2 & 26.1 & 31.2 \\
\hline rmsd bond length $(\AA)$ & & 0.012 & 0.005 & 0.006 & 0.006 \\
\hline rmsd bond angle $\left({ }^{\circ}\right)$ & - & 1.738 & 1.019 & 1.076 & 0.999 \\
\hline Ramachandran plot (\%) ${ }^{\mathrm{d}}$ & - & $92.5,7.0,0.5,0$ & $91.6,8.4,0,0$ & $92.0,7.9,0.2,0$ & $91.8,8.0,0.2,0$ \\
\hline
\end{tabular}

${ }^{a}$ Values in parentheses refer to data in the highest resolution shell.

$\left.{ }^{\mathrm{b}} R_{\mathrm{sym}}=\Sigma_{\mathrm{hkl}} \Sigma_{\mathrm{i}} l_{\mathrm{i}(\mathrm{hkl}}\right)-</(\mathrm{hkl})>\mid / \Sigma_{\mathrm{hkl}} \Sigma_{\mathrm{i}} l_{\mathrm{i}}(\mathrm{hkl})$.

${ }^{\mathrm{c}} R_{\text {work }}=\Sigma_{\mathrm{hkl}}\left|F_{\mathrm{o}}(\mathrm{hk})-F_{\mathrm{c}}(\mathrm{hk})\right| / \Sigma_{\mathrm{hkl}} F_{\mathrm{o}}(\mathrm{hk}) \cdot R_{\text {free }}$ was calculated with $5 \%$ of the data that were not used for refinement.

${ }^{\mathrm{d}}$ Percentage of residues in most favored, additional allowed, generously allowed and disallowed regions in Ramachandran plot.

the y-phosphate group of AMP-PNP molecule is found to be in a highly bent conformation (Fig. $2 \mathrm{E}$ ). The adenine groups of all nucleotide molecules are bound deep in a hydrophobic pocket within the cleft between the $\mathrm{N}$ - and C-lobes, while the tail groups are partially exposed to the solvent region. Superposition of all six nucleotide molecules (two molecules per dimeric MsStt7d-KD-nucleotide complex) indicates a fairly rigid adenine group and highly flexible polyphosphate groups with multiple conformations (Fig. 2E). When the phosphorylatable peptide substrate binds to the kinase, the terminal phosphate group will be fixed in an optimal orientation for the phosphoryltransfer reaction, as observed in the ternary complex structure of cAMP-dependent protein kinase, nucleotide and peptide substrates (Madhusudan et al., 1994).

\section{A novel hairpin motif stabilizes the activation loop in an} active conformation

Is the structure of MsStt7d-KD in an active or inactive state? To predict the conformational state of MsStt7d-KD in the crystal, the structure of MsStt7d-KD was compared with those of CDK2 in either active or inactive state (Schulze-Gahmen et al., 1996; Brown et al., 1999) (Fig. 3). The structure of MsStt7dKD closely resembles that of active-state CDK2 in two major aspects that are related to the activation of protein kinases (Endicott et al., 2012). Firstly, the $\alpha 1$ helix of MsStt7d-KD and the equivalent helix of active-state CDK2 (also named $C$ helix previously) are aligned in the same orientation, allowing the Glu residue (Glu 216 in MsStt7d-KD) to stabilize the Lys resi- 


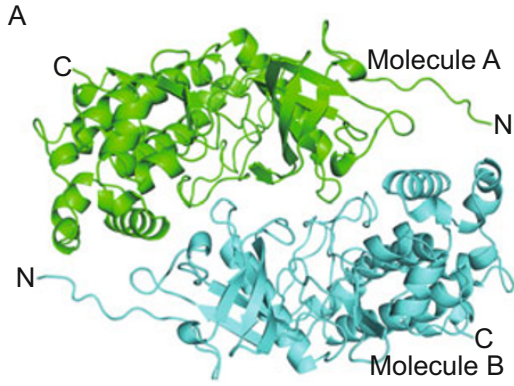

B

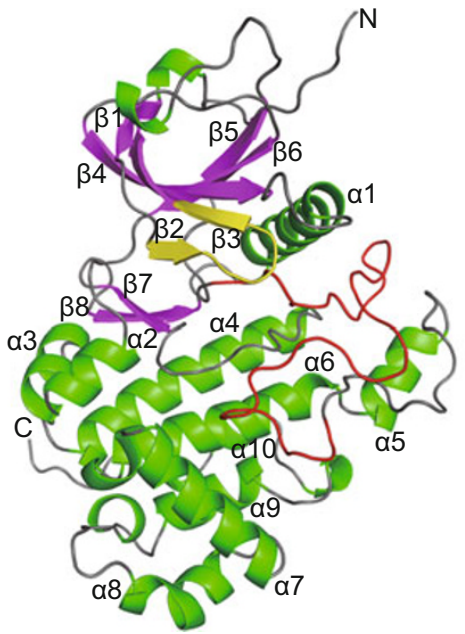

C

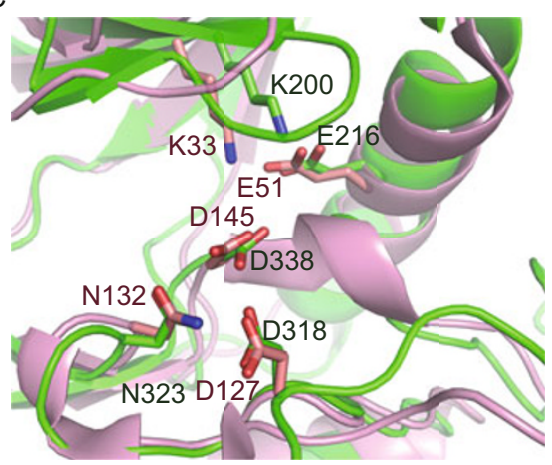

D

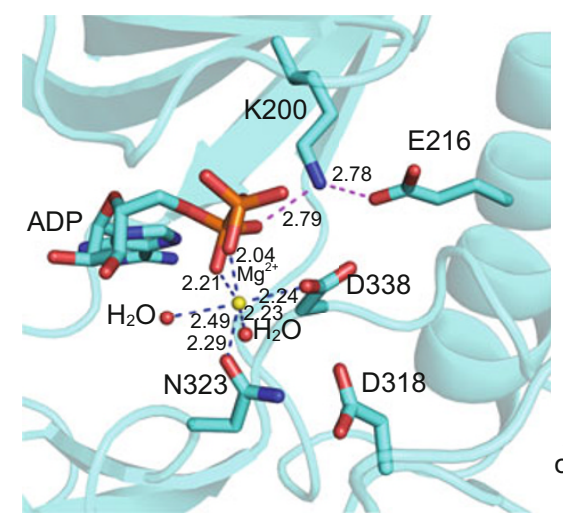

$\mathrm{E}$

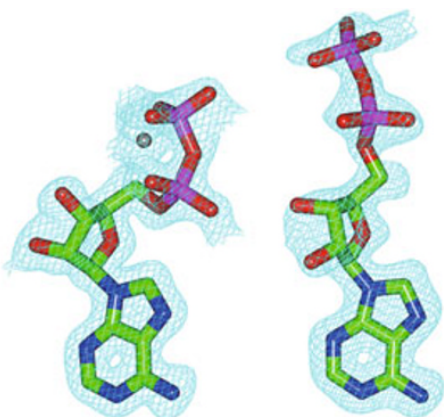

ADP in molecule A ATP (modeled as ADP) of MsStt7d-KD-ADP in molecule A of
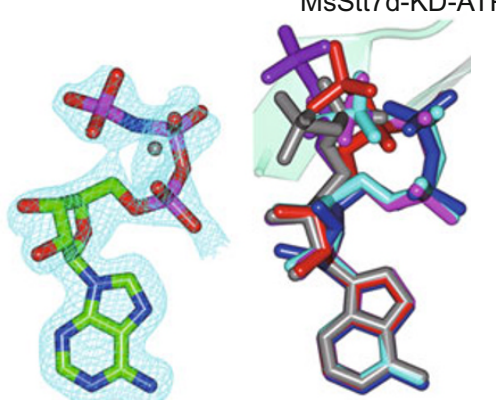

AMP-PNP in molecule A Six nucleotides from of MsStt7d-KD-AMP-PNP three complexes

Figure 2. Structures of MsStt7d-KD apoprotein and in complex with nucleotide substrates. (A) A dimer of the MsStt7d-KD apoprotein within the asymmetric unit. (B) The structure of monomeric MsStt7d-KD. The $\alpha$-helices are colored in green, the $\beta$-strands are in magenta and loops are in grey. The functionally important glycine-rich loop and activation loop regions are highlighted by yellow and red colors, respectively. (C) Conserved residues in the active site important for ATP binding and phosphoryl-transfer reaction. Green, MsStt7d-KD; pink, CDK2 (PDB code: 1QMZ). The labels for residues from MsStt7d-KD and CDK2 are in green and pink colors, respectively. (D) The ADP binding site of MsStt7d-KD-ADP complex structure. One ADP and one $\mathrm{Mg}^{2+}$ per monomer are assigned in the complex structure. The blue dashes indicate the coordination bonds around $\mathrm{Mg}^{2+}$; the magenta dashes indicate the ionic interactions between K200 and E216 or ADP a-phosphate group. The numbers near the dash lines show the distances between two atoms $(\AA)$. (E) $2 F_{\mathrm{o}}-F_{\mathrm{c}}(1.0$ $\sigma$ level) electron densities of ADP, ATP and AMP-PNP molecules bound to MsStt7d-KD and superposition of all six nucleotide molecules observed in the complex structures. The ATP molecules in the MsStt7d-KD-ATP complex structures have no detectable features for the Y-phosphate groups in the electron density map (presumably due to hydrolysis by the kinase) and thereby modeled as ADP molecules instead.

due (Lys 200 in MsStt7d-KD) in a position optimal for the binding of ATP a-phosphate group (Figs. 2D and 3A). Secondly, the activation loops of MsStt7d-KD and active-state CDK2 are both positioned beside the catalytic cleft to form a polypeptide substrate-binding platform on one hand and open up the active site for the access of ATP molecule on the other hand (Fig. 3A). In comparison, the inactive-state CDK2 has a tilted $C$ helix and its conserved Glu and Lys residues drift away from the ATP binding site. Moreover, the catalytic cleft in the inactive-state CDK2 is obstructed by the activation loop (Fig. 3B). Therefore, the structure of MsStt7d-KD is most likely in an active conformation ready for accepting substrates and performing catalysis.

In the case of CDK2, when the activator, cyclin A, binds to the $C$ helix and the activation loop region, the $C$ helix is reoriented and the activation loop moves away from the catalytic cleft, leading to the activation of CDK2 (Jeffrey et al., 1995). Why is the MsStt7d-KD trapped in an active state? A novel hairpin motif was found adjacent to the activation loop region of MsStt7d-KD (Figs. 3 and 4A). This motif consists of the $\alpha 5$ helix (Ala 387-Pro 395) and a short loop (Pro 376-Ala 386) antiparallel to the a5 helix, and is named a5 hairpin hereby. The a5 hairpin forms extensive hydrophobic interactions and hydrogen bonds with residues from the activation loop region. On the hairpin surface which contacts the activation loop, two hydrophobic pockets accommodate the side chains of Tyr 354 and Pro 356 from the activation loop region, respectively (Fig. 4B). Furthermore, the residues Ala 386, Ala 387, Trp 390 and Pro 395 from a5 hairpin form extensive hydrophobic interactions with residues Cys 345, Leu 346 and Pro 347 from the activation loop, enhancing the interactions between the two regions (Fig. 4B). Besides, water molecules located at the 
A

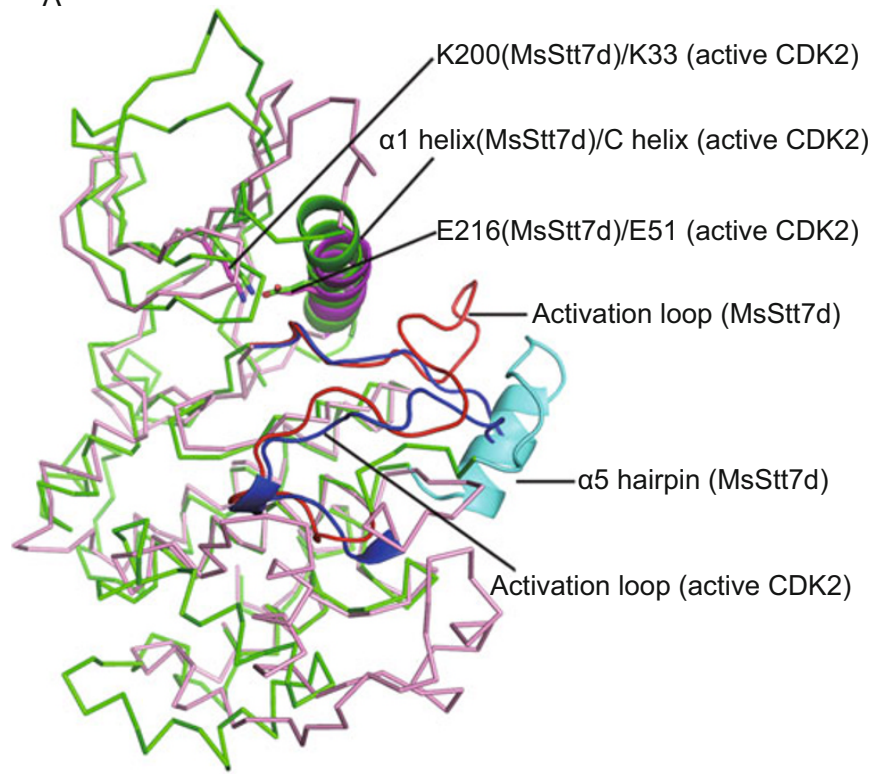

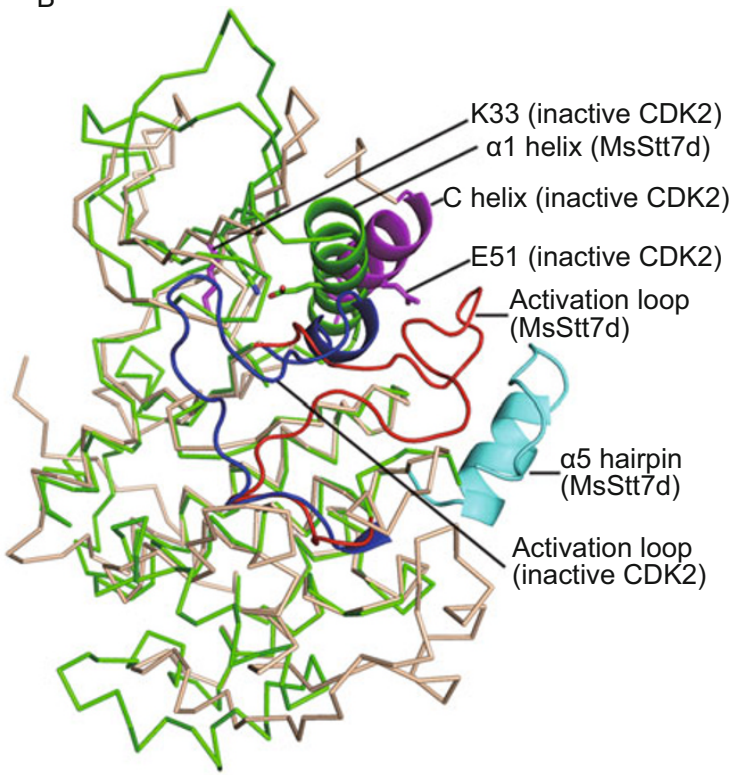

Figure 3. Superposition of MsStt7d-KD structure with that of CDK2 in the active or inactive state. (A) Superposition of MsStt7d-KD (green) with the active-state CDK2 (PDB code: 1QMZ, pink). The $\alpha 1$ helix (green), activation loop (red) and a5 hairpin (cyan) of MsStt7d$\mathrm{KD}$, and the $\mathrm{C}$ helix (magenta) and the activation loop (blue) of active CDK2 are shown as cartoon models. The side chains of the key residues Lys and Glu from two molecules are shown as stick models. (B) Superposition of MsStt7d-KD (green) with the inactive-state CDK2 (PDB code: $1 \mathrm{HCL}$, wheat). The $\mathrm{C}$ helix, the activation loop, the key residues Lys and Glu of inactive CDK2 are shown in the same colors as those of active CDK2 in (A).

interface between the activation loop and the hairpin contribute to the formation of hydrogen bonds which further strengthen the interactions between the two regions (Fig. 4C). Thus, the activation loop is fixed in a conformation which closely resembles that of the active-state CDK2 (Brown et al., 1999) (Fig. 3A), but differs dramatically from that of the inactive-state CDK2 (Schulze-Gahmen et al., 1996) (Fig. 3B). When the a5 helix was deleted from the MsStt7d-KD protein (the $\Delta 386-393$ mutant), the majority of target protein ended in the insoluble fraction when expressed in E. coli, suggesting that the deletion mutant was highly unstable or did not fold properly. According to the sequence alignment result, the a5 hairpin is a conserved feature also present in other Stt7/STN7 family members, but absent in CDK2 or other protein kinases (Fig. 4D). Although it appears that the seven residues between Pro 381 and Leu 389 in MsStt7d are not well conserved when compared to the other Stt7/STN7 members, a close examination on the structure reveals that the side chains of these residues point away from the interface between the $\alpha 5$ hairpin and the activation loop. They are exposed on the protein surface, thus explaining why the sequence of this region is relatively variable. Furthermore, sequence-based prediction on the secondary structures of CrStt7/AtSTN7 indicates that their corresponding regions aligning to the a5 hairpin of MsStt7d form similar coil-helix structures. Therefore, the hairpin motif containing a " $\mathrm{PX}_{7 / 8} \mathrm{LW}$ " consensus sequence (Fig. 4D) is a characteristic feature for the Stt7/STN7 family which not only has an important role in stabilizing the activation loop in the active state, but also is essential for protein stability.

\section{MsStt7d-KD exhibits basal autophosphorylation activity toward both Thr and Tyr residues}

To further analyze whether the MsStt7d-KD protein is active in converting ATP to ADP in solution, a luminescent ADP-detection assay was performed on the wild-type, K200R, D318A and D318N mutant proteins. The three active-site mutants had nearly abolished activity in comparison with the wild-type protein (Fig. 5A). Evidently, residues Lys 200 and Asp 318 are essential for its ADP-producing activity. As no exogenous peptide substrates were added in the luminescent ADP-detecting assay, the activity of wild-type MsStt7d-KD is therefore attributed to the intrinsic ATPase activity and/or autophosphorylation activity. When the kinase was incubated with radioactive substrate $\left[\mathrm{Y}^{-}{ }^{32} \mathrm{P}\right]$ ATP, we found that the purified MsStt7d-KD protein displayed basal autophosphorylation activity (Fig. 5B). The active-site mutants including K200R, D318A and D318N all lost their autophosphorylation activity (Fig. 5B). Previous in vivo study showed that the K167R and K167Q mutants of AtSTN7 had abolished function in state transition (Bellafiore et al., 2005). Lys 200 in MsStt7d is the equivalent residue of Lys 167 in AtSTN7. Therefore, the abolished kinase activity of the MsStt7d-KD K200R mutant observed through our in vitro assays is consistent with the loss-of-function phenotype of 
A

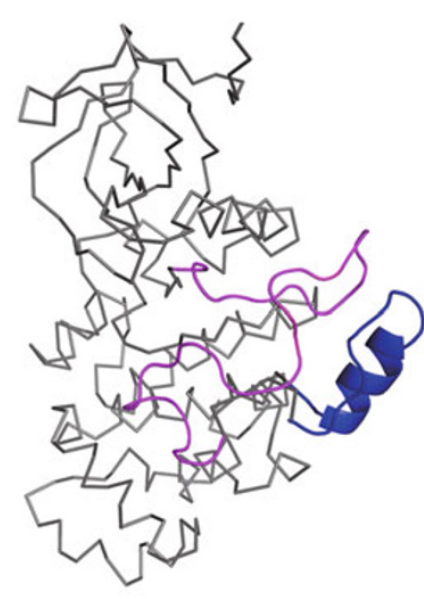

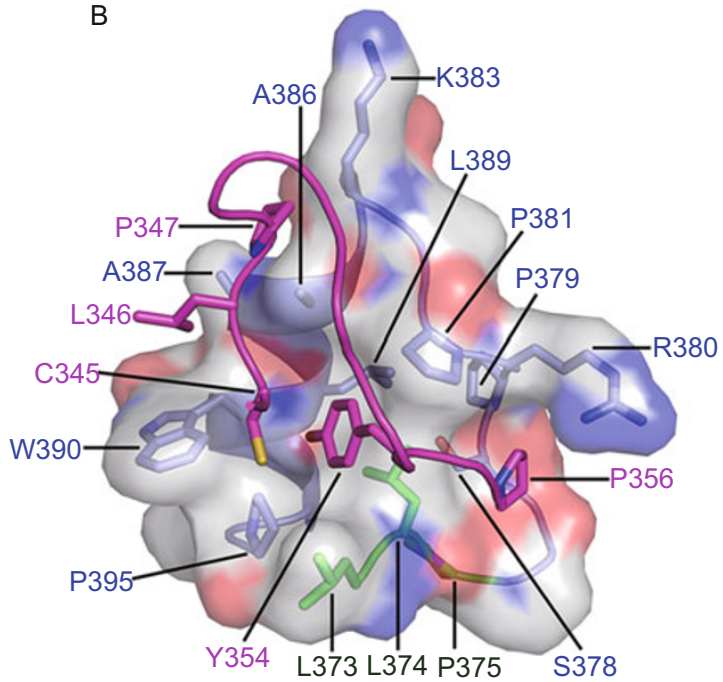

C

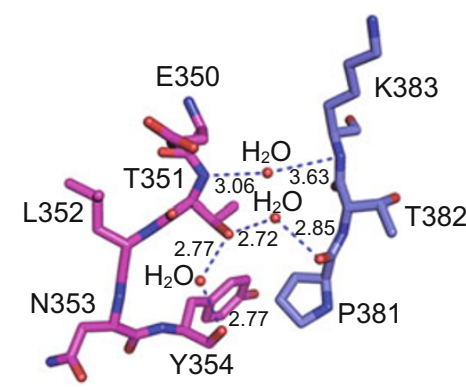

D

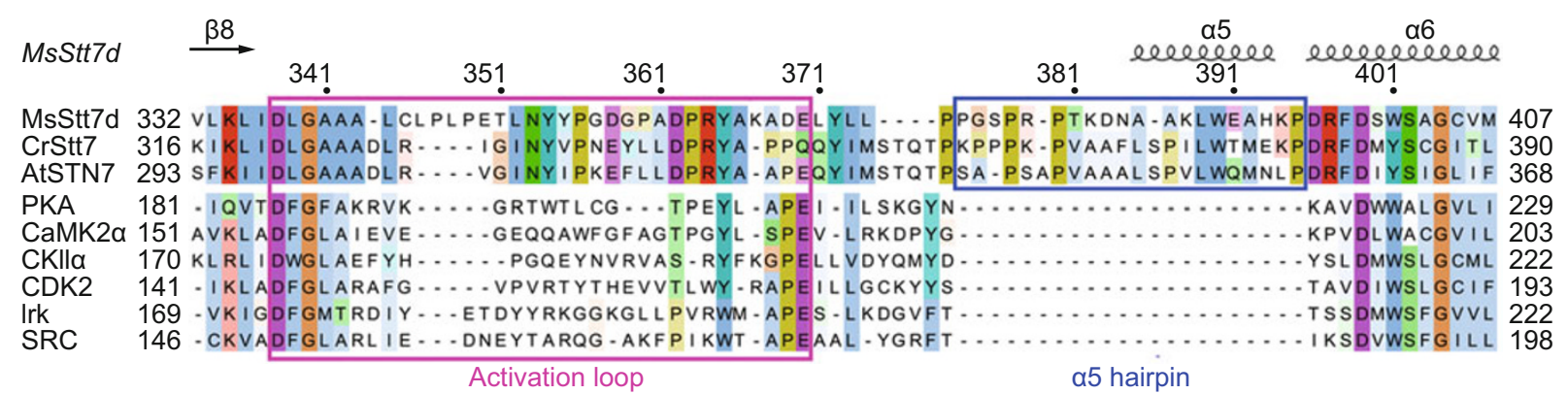

Figure 4. A novel hairpin motif discovered in the structure of MsStt7d-KD. (A) The a5 hairpin motif is positioned next to the activation loop. The activation loop (magenta) and the $a 5$ hairpin (blue) are shown as cartoon models. (B) The interactions between the $\alpha 5$ hairpin and the activation loop. The $\alpha 5$ hairpin is shown as a cartoon model superposed with a semi-transparent surface presentation. Amino acid residues involved in the hydrophobic interactions at the a5 hairpin-activation loop interface are highlighted as stick models. Color codes: blue, residues from the a5 hairpin; magenta, residues from the activation loop; green, residues from other regions. (C) Hydrogen bond interactions bridged by water molecules at the a5 hairpin-activation loop interface. The numbers near the dash lines show the lengths of the hydrogen bonds $(\AA)$. (D) Sequence alignment showing that other members of Stt7/STN7 family (CrStt7 and AtSTN7) also contain stretches of amino acid sequences similar to that of the $\alpha 5$ hairpin motif in MsStt7d. Other protein kinases are included as negative controls showing the absence of such a feature in their sequences. Abbreviations: PKA, cAMP-dependent protein kinase catalytic subunit $\alpha$ isoform 1 from Homo sapiens; CaMK2a, calcium/calmodulin-dependent protein kinase type II subunit $\alpha$ isoform 1 from Homo sapiens; CKIla, casein kinase II subunit a from Mus musculus; CDK2, cyclin-dependent kinase 2 from Homo sapiens; Irk, Tyr kinase domain of insulin receptor from Homo sapiens; SRC, Tyr protein kinase C-Src from Homo sapiens.

\section{K167R/K167Q mutant of AtSTN7.}

The autophosphorylation reaction products of MsStt7d-KD were analyzed through western blots with anti-phosphoserine, anti-phosphothreonine or anti-phosphotyrosine antibody to identify which type/types of amino acid residues on the protein was/were phosphorylated. The results indicate that the dominating autophosphorylation sites in MsStt7d-KD are on Thr and Tyr residues, but not Ser residues (Fig. 5C). Subsequently, identification of the phosphorylation sites using mass spectrometry has led to the finding that MsStt7d-KD is primarily autophosphorylated on residue Thr 185 in the glycine-rich loop and Tyr 221 in the a1 helix (Fig. 5D and 5E). Further analysis on the autophosphorylation level of T185A and Y221F mutants (Fig. 5F) indicates that Thr 185 is the major autophosphoryla- tion site, while Tyr 221 is a minor site. The results demonstrate that MsStt7d-KD exhibits basal autophosphorylation activity toward both Thr and Tyr residues. Thus, MsStt7d-KD belongs to the dual-specificity protein kinase family which is capable of phosphorylating both Thr and Tyr residues (Lindberg et al., 1992).

\section{The peptide substrate binding site of MsStt7d-KD}

In order to test whether MsStt7d-KD is capable of phosphorylating the $\mathrm{N}$-terminal peptides of the light-harvesting complexes directly, we have constructed, expressed and purified various fusion proteins with the RKXVA pentapeptide (X being T/Y for phosphorylation) fused to MsStt7d-KD at the $\mathrm{N}$-terminus. The RKTVA is a consensus $\mathrm{N}$-terminal sequence shared between 
A

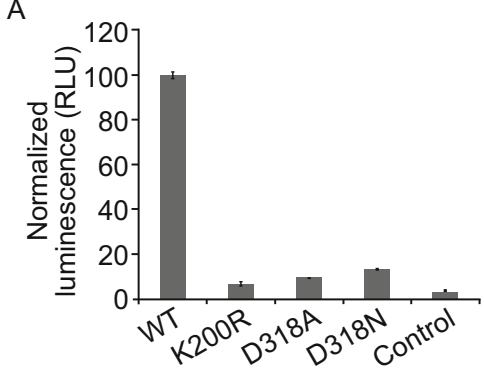

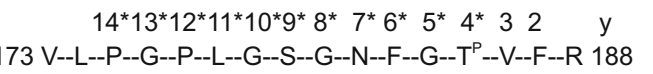

b

$\begin{array}{llllllllll}4 & 5 & 6 & 7 & 8 & 9 & 10 & 11 & 12 & 13^{*} 14^{*} 15^{*}\end{array}$

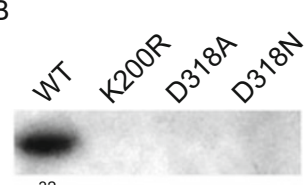

${ }^{32} \mathrm{P}$-Autoradiogram

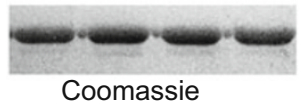

E b
C

P-Ser antibody P-Thr antibody P-Tyr antibody KD P-KD KD P-KD KD P-KD

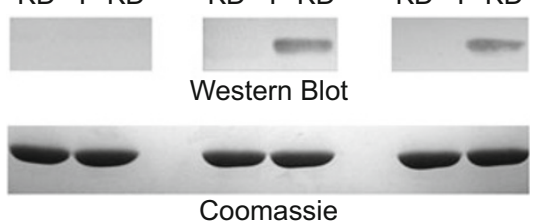

Coomassie
$\mathrm{F}$
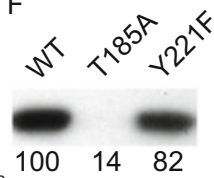

${ }^{32}$ P-Autoradiogram

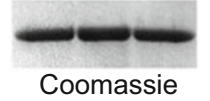

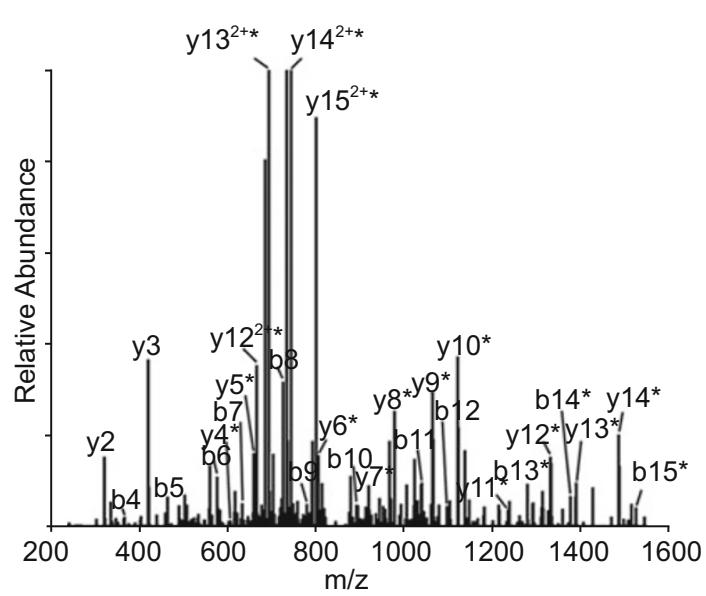

$24^{*} 23^{*} 22^{*} 21^{*} 20^{*} 19^{*} 18^{*} 17^{*} 16^{*}$

$8^{*} \quad \begin{array}{llll}6 & 5 & 4 & 3\end{array}$ $\mathrm{y}$ 204 A--D--V--M\#--A--A--E--E--L--L--E--C--E--M\#--D--V--N--YP--H--V--H--A--N--A--K 228 678

$17 \quad 19^{*} \quad 23^{*}$

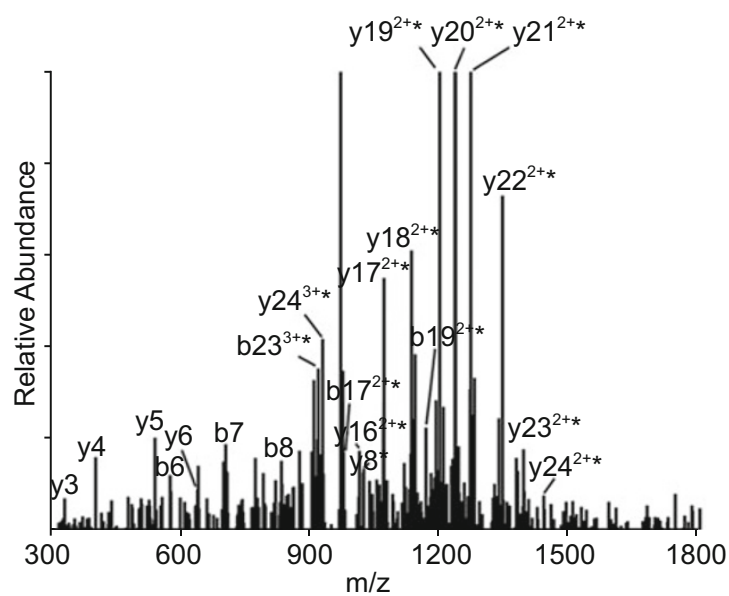

Figure 5. Activity assays on MsStt7d-KD and its mutants. (A) The kinase activity of wild-type (WT) and active-site mutants of MsStt7dKD measured through luminescent ADP-Glo assay (Promega). The activity data is present as the normalized relative light unit (RLU) of luminescence signal correlated to the amount of ADP produced by the kinase. The data are the mean values of three independent experiments \pm standard deviations (SDs). (B) Autophosphorylation of the wild-type MsStt7d-KD and active-site mutants as detected by autoradiography using $\left[\mathrm{Y}^{-}{ }^{32} \mathrm{P}\right]$ ATP as the kinase substrate. The gels stained with Coomassie Brilliant Blue are shown as loading controls in all the figures with "Coomassie" labels throughout the paper. (C) Western blots analysis of the autophosphorylation products using antiphosphoserine, anti-phosphothreonine and anti-phosphotyrosine antibodies, respectively. The "KD" and "P-KD" indicate the MsStt7d-KD samples before and after autophosphorylation reaction, respectively. (D) The MS/MS spectrum of a doubly charged ion at $\mathrm{m} / \mathrm{z}=849.53$ for $\mathrm{MH}_{2}{ }^{2+}$ corresponding to the mass of the phosphorylated peptide VLPGPLGSGNFGTPVFR (173-188). The * labeled peaks correspond to masses of $b$ and $y$ ions of phosphorylated peptide fragments. ( $E$ ) The MS/MS spectrum of a triply charged ion at $\mathrm{m} / \mathrm{z}=992.05$ for $\mathrm{MH}_{3}{ }^{3+}$ corresponding to the mass of the phosphorylated and oxidized peptide ADVM\#AAEELLECEM\#DVNYPHVHANAK (204-228). The * labeled peaks correspond to masses of $b$ and $y$ ions of phosphorylated peptide fragments; the \# indicates the oxidized methionine residues. (F) Verification of the MS-detected autophosphorylation sites through mutagenesis and autoradiography. The relative integrated intensity over the area of each band is shown below autoradiogram.

the light harvesting proteins Lhcb1 from Arabidopsis thaliana (AtLhcb1) and the Lhcp4 from Micromonas sp. RCC299 (MsLhcp4). Compared with the wild-type MsStt7d-KD, the phosphorylation level of RKTVA-MsStt7d-KD increased dramatically (Fig. 6A). The RKYVA-MsStt7d-KD protein also displayed enhanced phosphorylation at a level even higher than that of RKTVA-MsStt7d-KD. In contrast, when the residue Thr/ Tyr was mutated to Ser/Ala/Phe, the phosphorylation of these three fusion proteins (RKSVA-MsStt7d-KD, RKAVA-MsStt7dKD and RKFVA-MsStt7d-KD) decreased to a level close to that of the wild-type protein (Fig. 6A). Therefore, the observed en- hanced phosphorylation of RKTVA-MsStt7d-KD and RKYVAMsStt7d-KD is most likely due to direct phosphorylation on the exogenous peptides fused to the N-terminus of MsStt7d-KD. Consistent with the observed basal autophosphorylation on endogenous Thr and Tyr residues (Fig. 5), MsStt7d-KD may also phosphorylate exogenous $\mathrm{Thr}$ and Tyr residues covalently linked to the $\mathrm{N}$-terminal region of the kinase domain (Fig. 6A).

To gain insights into the peptide/protein substrate binding site on MsStt7d-KD, the structure of CDK2-peptide complex (Brown et al., 1999) or insulin receptor kinase (Irk, a tyrosine kinase)-peptide complex (Hubbard, 1997) is superposed onto 
A

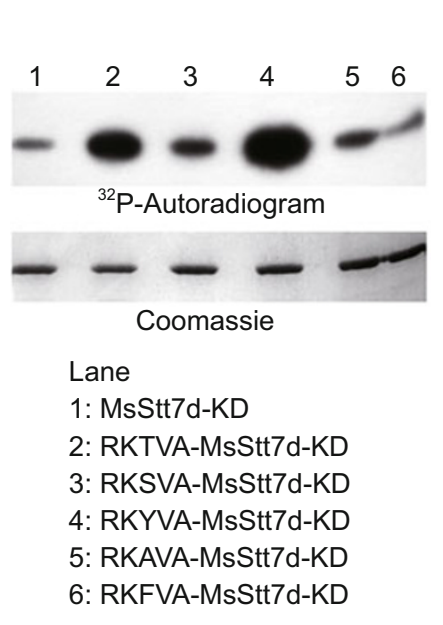

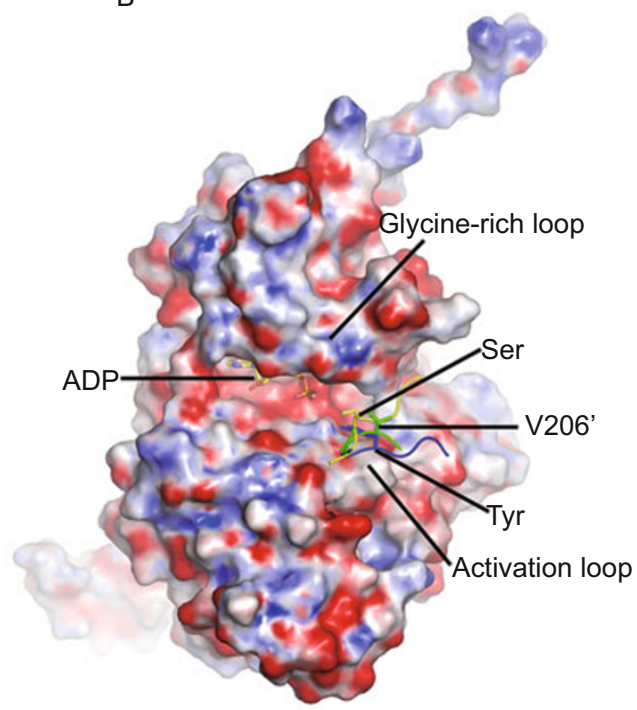

C

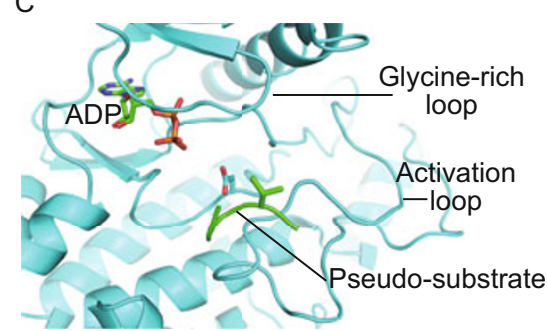

D

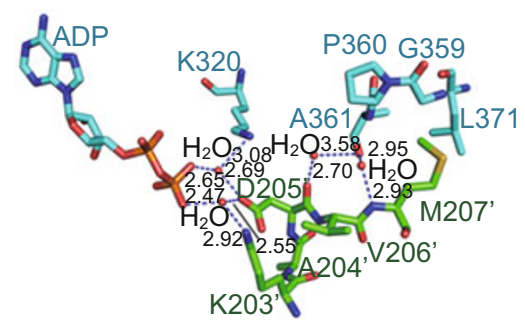

Figure 6. The potential peptide substrate binding site of MsStt7d-KD. (A) The phosphorylation activity of MsStt7d-KD toward the "RKXVA" (X being T/S/Y/A/F) pentapeptides covalently fused to its $\mathrm{N}$-terminus. The "RKTVA" pentapeptide is a consensus $\mathrm{N}$-terminal sequence shared by Micromonas Lhcp4 and Arabidopsis Lhcb1. (B) The MsStt7d-KD is shown as electrostatic potential surface model with calculated electrostatic potential in the range from $-15 \mathrm{kT} / \mathrm{e}$ (deep red, electronegative) to $15 \mathrm{kT} / \mathrm{e}$ (deep blue, electropositive). $\mathrm{k}$, the Boltzmann constant; T, the temperature; e, the magnitude of the electron charge. ADP molecule is shown as a stick model. The yellow and blue peptides are the substrates of CDK2 (PDB code: 1QMZ) and Irk (PDB code: 1IR3), respectively. They are docked on the surface of MsStt7d-KD by superposing CDK2 and Irk structures (omitted from the figure for clarity) with the MsStt7d-KD structure. The green peptide is the pseudo-substrate from the $\beta 4-\alpha 1$ loop of the adjacent molecule of MsStt7d-KD in the crystal. (C) A zoom-in view of the pseudo-substrate binding site of MsStt7d-KD. MsStt7d-KD is shown in cyan; the pseudo-substrate polypeptide is colored in green. D318 and V206' indicate key residues from the MsStt7d-KD enzyme and pseudo-substrate, respectively. (D) Interactions between the pseudosubstrate (green) polypeptide and MsStt7d-KD (cyan). The hydrogen bonds are indicated by the blue dashes. The numbers near the dash lines show the distances between two adjacent atoms forming hydrogen bonds $(\AA)$. The residues from pseudo-substrate are labeled with ' symbols.

that of MsStt7d-KD-ADP complex. The result shows that the potential binding site for the peptide substrate on MsStt7d$\mathrm{KD}$ is around the flat surface area between the glycine-rich loop and activation loop region (Fig. 6B). The a-carbon atom of Tyr residue on the Irk substrate peptide moves outwards by $4.9 \AA$ as compared with the $\alpha$-carbon atom of Ser residue on the CDK2 substrate peptide. The terminal hydroxyl groups of both Ser and Tyr residues are located merely $3.6 \AA$ and $3.3 \AA$ from the side chain of the catalytic residue Asp 318 in MsStt7d$K D$, suggesting that they are in a position ready to accept the Y-phosphate group from the ATP molecule.

A straightforward way of observing the interactions between the peptide substrates and MsStt7d-KD is to solve the structures of MsStt7d-KD in complex with peptide substrates. Extensive attempts have been made either by soaking the MsStt7d-KD crystals in a solution with excess peptide substrates or by co-crystallizing the MsStt7d-KD with peptide substrates. Nevertheless, no peptide substrates were found in the structures solved, indicating the binding site might not be accessible to the peptide substrates within the crystal. Further analysis on the structure of MsStt7d-KD-ADP complex reveals the presence of a pseudo-substrate occupying the potential peptide binding site. In the dimer of MsStt7d-KD-ADP complex within the asymmetric unit, residue 203-207 (with the sequence of KADVM) on the $\beta 4-\alpha 1$ loop from one monomer binds to the potential peptide substrate binding site of the other monomer (Fig. 6C), thereby mimicking the substrate-bound state (Fig. 6B). In this pseudo-substrate, the phosphorylatable residue has been replaced by a non-phosphorylatable Val residue (Val 206' with the ' symbol indicating that the residue is from the adjacent molecule) whose side chain points away from the active site. The interactions between MsStt7d-KD and its pseudo-substrate peptide are mainly hydrogen bonds between Asp 205'/Lys 203' and the water molecules surrounding the $\beta$-phosphate group of ADP, hydrogen bonds between Asp 205'/Met 207' and Ala 361 bridged by water molecules, and hydrophobic interactions between Met 207' and Gly 359/ Ala 361/Leu 371 (Fig. 6D). Coincidently, the pseudo-substrate binding site lies between the predicted binding sites for Ser/ Thr-containing peptide and Tyr-containing peptide (Fig. 6B), which further supports that this area is most likely for the binding of polypeptide/protein substrates.

As shown in Fig. 6B, it is predictable that the position of peptide backbone docking site might be adjustable on the flat 
surface of MsStt7d depending on the size of phosphorylatable residue, so that the terminal hydroxyl group can be positioned close enough to both the catalytic residue (Asp 318) and the ATP $y$-phosphate for phosphorylation reaction to occur (Fig. 6B). The open and flat surface feature of the peptidebinding site might account for the capability of MsStt7d-KD in phosphorylating both Thr and Tyr residues.

\section{DISCUSSION}

When the MsStt7d protein sequence is used to search the RefSeq protein database of Arabidopsis thaliana for its potential homologs through the BLASTP program (Altsch et al., 1997), the result shows top two hits being STN7 and STN8 with Evalues of $2 \times 10^{-38}$ and $3 \times 10^{-43}$, respectively. STN8 is a close homolog of STN7 which is essential for the phosphorylation of photosystem II core proteins (Bonardi et al., 2005). Since MsStt7d does not contain a long STN8-specific loop inserted between the $\beta 3$ and $\beta 4$ strands (Vainonen et al., 2005), it is evident that MsStt7d resemble STN7 instead of STN8. Unlike CrStt7 or AtSTN7 which has a predicted N-terminal transmembrane helix and a pair of lumen-exposed Cys residues, the full-length MsStt7d has a predicted PDZ domain fused to the kinase domain at the $\mathrm{N}$-terminal region instead. The presence of a PDZ domain at the $\mathrm{N}$-terminal region of MsStt7d might provide an alternative mechanism for kinase activity regulation. Nevertheless, the BLAST result strongly suggests that MsStt7d is a homolog of the station transition kinase family, but with a different $\mathrm{N}$-terminal region.

Through structural analysis of MsStt7d-KD, we have discovered that the canonical protein kinase domain and the newly identified a5 hairpin motif are both the conserved features of Stt7/STN7 family (Fig. 1). The MsStt7d-KD structure reported here serves as the homologous model of the core domain of Stt7/STN7. Based on the following observation, we believe the structure of MsStt7d-KD represents the kinase domain of Stt7/STN7 in the active but autoinhibited state. Firstly, the structure of MsStt7d-KD closely resembles that of CDK2 in the active state (Brown et al., 1999) (Fig. 3) whose activation loop swings away from the active site, favoring the binding of both nucleotide and peptide substrates. Secondly, the MsStt7d-KD dimer observed in the crystal has its peptide binding sites occupied by the pseudo-substrates from the adjacent molecules (Fig. 6C), suggesting that the kinase is in an active but autoinhibited state when it forms a dimer.

Sequence alignment of various Stt7/STN7 homologs shows that $\mathrm{CrStt} 7$ contains a phosphorylatable Thr residue in the glycine-rich loop region equivalent to Thr 185 (confirmed autophosphorylation site) in MsStt7d (Fig. 1). The relative conservation of phosphorylatable site in the glycine-rich loop may imply a potential role of this site for the regulation of kinase activity. In CDK2, residues Tyr 15 and Thr 14 in the glycinerich loop are phosphorylated by other kinases, and their phosphorylations negatively regulate the catalytic activity of CDK2 through reducing the affinity of kinase toward the peptide/ protein substrate (Welburn et al., 2006). Recently, autophosphorylation within the glycine-rich loop of Brassinosteroid insensitive 1 (BRI1) receptor kinase from Arabidopsis thaliana has been reported to reduce the kinase activity of BRI1 too (Oh et al., 2012). Therefore, it is likely that autophosphorylation at the glycine-rich loop in Stt7 may also have a similar regulatory effect on the kinase activity.

It was reported earlier that the C-terminal region of STN7 contained four phosphorylation sites (Ser 526, Thr 537, Thr 539, and Thr 541) identified through a large-scale Arabidopsis phosphoproteome profiling study (Reiland et al., 2009). A follow-up work suggested that phosphorylation of STN7 on these sites might have a role in regulating its turnover during state transition, while loss of the phosphorylation sites did not affect its function in state transition (Willig et al., 2011). For both studies, the phosphorylation of STN7 was not assigned as a consequence of autophosphorylation activity due to the complexity of in vivo system when many other protein kinases are present (Reiland et al., 2009; Willig et al., 2011). By using the in vitro assay system with highly purified MsStt7d-KD kinase sample, we are now able to demonstrate that the Micromonas Stt7d homolog exhibits autophosphorylation behavior. Our data suggest a possibility that autophosphorylation might be responsible, at least partly, for the phosphorylation of STN7 under in vivo conditions.

Previous studies suggested that STN7 may have important roles in LTR in addition to its well-established role in state transition (Bonardi et al., 2005; Wagner et al., 2008; Pesaresi et al., 2009). It is proposed that STN7 senses the redox signal of $P Q$ pool and transduces it to the level of gene expression in chloroplast and nucleus (Bonardi et al., 2005; Rochaix, 2007; Dietzel et al., 2008; Wagner et al., 2008; Pesaresi et al., 2009; Lemeille and Rochaix, 2010). The discovery of dual-substrate specificity of MsStt7d opens a new avenue for searching downstream Thr/Ser and/or Tyr-containing substrate proteins of STN7 kinases involved in the signaling process during LTR. Although it remains to be verified if the other STN7 members are also dual-specificity kinases, such a property might account for the versatile functions of STN7 in photosynthetic acclimation.

\section{MATERIALS AND METHODS}

Protein expression, purification and crystallization

The gene encoding MsStt7d kinase domain (Asp 140-Asp 489) was ligated into pET28a vector (Novagen) between Nco I and Not I sites. Mutants were constructed using the QuikChange Site-Directed Mutagenesis method (Agilent). For protein fused with pentapeptide substrate, the DNA sequence encoding the pentapeptide RKXVA (X being T/S/Y/A/F) was introduced to the 5 '-end of MsStt7d-KD gene. The final constructs were confirmed by DNA sequencing. The vectors were transformed into $E$. coli BL21 (DE3) and protein expression was induced at $20^{\circ} \mathrm{C}$ with $0.4 \mathrm{mmol} / \mathrm{L}$ IPTG when the cell density reached an $\mathrm{OD}_{600 \mathrm{~nm}}$ of $0.6-0.8$. Protein was purified through immobilized metal ion affinity chromatography (IMAC) using Nickel-Chelating Sepharose 
Fast Flow (GE Healthcare), followed by size exclusion chromatography using Superdex 200 10/300 GL (GE Healthcare) with a buffer containing $20 \mathrm{mmol} / \mathrm{L}$ Tris $\mathrm{pH} 8.0$ and $200 \mathrm{mmol} / \mathrm{L} \mathrm{NaCl}$. The eluted major peak fraction was concentrated to $10 \mathrm{mg} / \mathrm{mL}$ protein and used for crystallization trials. The best crystals of MsStt7d-KD were grown at $18^{\circ} \mathrm{C}$ using hanging-drop vapor diffusion method by mixing $1 \mu \mathrm{L}$ of protein sample and $1 \mu \mathrm{L}$ of well solution containing $0.1 \mathrm{~mol} / \mathrm{L}$ HEPES $\mathrm{pH} 7.5$, $200 \mathrm{mmol} / \mathrm{L} \mathrm{MgCl}_{2}$ and 25\% PEG3350. The MsStt7d-KD-nucleotide complex crystals were prepared by soaking the MsStt7d-KD crystals overnight in an artificial mother liquor (0.1 mol/L HEPES pH 7.5, 200 $\mathrm{mmol} / \mathrm{L} \mathrm{MgCl}_{2}, 27 \%$ PEG3350) supplemented with $10 \mathrm{mmol} / \mathrm{L}$ nucleotide substrate (ADP/ATP/AMP-PNP).

\section{Data collection and structure determination}

For phasing, a high-quality MsStt7d-KD crystal was soaked briefly in an iodide-containing derivatizing solution $(0.1 \mathrm{~mol} / \mathrm{L}$ HEPES pH 7.5, $200 \mathrm{mmol} / \mathrm{L} \mathrm{MgCl} 2,27 \%$ PEG3350 and $1 \mathrm{~mol} / \mathrm{L} \mathrm{KI}$ ) for $45 \mathrm{~s}$ at room temperature and then flash frozen in a $100 \mathrm{~K}$ cold nitrogen gas stream. A high-redundancy set of diffraction data of the MsStt7d-KD-KI derivative crystal were collected on a MicroMax 007 rotation-anode X-ray source with Rigaku Raxis IV++ image plate area detector. The data for the native MsStt7d-KD and complexes with various nucleotide substrates were collected at BL17U of Shanghai Synchrotron Radiation Facility with ADSC Q315r CCD detector. Data were processed and scaled with iMOSFLM (Battye et al., 2011) and Scala (Evans, 2006) or with the HKL2000 package (Otwinowski and Minor, 1997). The initial phases were solved by the single wavelength anomalous dispersion (SAD) method using the MsStt7d-KD-KI derivative data with the AutoSol program in the PHENIX software suite (Adams et al., 2010). In the asymmetric unit, a total of twenty three iodide atoms with occupancy above 0.20 were located. The figure of merit (FOM) of the initial phases was 0.39 which was further improved to 0.68 after density modification. Around $90 \%$ of the structural model was automatically built by the AutoBuild program in PHENIX suite (Adams et al., 2010). The output model was corrected and readjusted manually in the COOT program (Emsley and Cowtan, 2004). The structures were refined using CNS (Brunger et al., 1998), Phenix.refine (Adams et al., 2010) and Refmac (Murshudov et al., 1997) in the CCP4 package (CCP4, 1994). The structures of native MsStt7d-KD and in complex with nucleotide substrates were solved by the difference Fourier method using the structure of MsStt7d-KD-KI derivative as the initial model. The final refined structures were checked with program PROCHECK (Laskowski et al., 1993). Structural figures were prepared using PyMOL (DeLano, 2002) or CCP4MG (McNicholas et al., 2011). Data collection and structure refinement statistics is summarized in Table 2.

\section{ADP-Glo kinase assay}

ADP-Glo kinase assay was performed as described in the protocol (Promega). Each reaction mixture contained $2 \mu \mathrm{mol} / \mathrm{L}$ kinase and $100 \mu \mathrm{mol} / \mathrm{L}$ ultrapure ATP in $25 \mu \mathrm{L}$ of reaction buffer $(40 \mathrm{mmol} / \mathrm{L}$ Tris $\mathrm{pH} 7.5$, $20 \mathrm{mmol} / \mathrm{L} \mathrm{MgCl}_{2}$ and $0.1 \mathrm{mg} / \mathrm{mL} \mathrm{BSA}$ ). The reaction was initiated by adding ultrapure ATP, and after incubation at $20^{\circ} \mathrm{C}$ for $1 \mathrm{~h}$ it was terminated by adding equal volume of ADP-Glo Reagent. After incubation at room temperature for $40 \mathrm{~min}$ to deplete the remaining original ATP, $50 \mu \mathrm{L}$ of Kinase Detection Reagent was added to convert ADP into ATP and the newly synthesized ATP was detected through the luciferase/ luciferin reaction which produced luminescence signal. After incubation at room temperature for $60 \mathrm{~min}$, luminescence was recorded using Varioskan Flash Multimode Reader (Thermo). The control experiment was carried out under the same reaction condition without adding the kinase. For each reaction, three repeats were performed for the calculation of mean values and standard deviations (SDs).

\section{Kinase autophosphorylation assay and autoradiography}

The phosphorylation reaction mixture $(25 \mu \mathrm{L}$ volume) contained $20 \mathrm{mmol} / \mathrm{L} \mathrm{HEPES}, \mathrm{pH} 7.5,50 \mathrm{mmol} / \mathrm{L} \mathrm{NaCl}, 10 \mathrm{mmol} / \mathrm{L} \mathrm{MgCl}_{2}$, $10 \mathrm{mmol} / \mathrm{L} \mathrm{NaF}, 0.05 \% \mathrm{n}$-Dodecyl- $\beta$-D-Maltoside, $4 \mu \mathrm{mol} / \mathrm{L}$ kinase, $0.4 \mathrm{mmol} / \mathrm{L}$ ATP and $10 \mu \mathrm{Ci}\left[\mathrm{Y}^{-}{ }^{32} \mathrm{P}\right]$ ATP. The phosphorylation reaction was initiated by the addition of ATP, and after incubation at room temperature for $2 \mathrm{~h}$ the reaction was terminated by adding SDS-PAGE sample loading buffer. The sample was heated at $100^{\circ} \mathrm{C}$ for $10 \mathrm{~min}$ and then loaded onto the gel for SDS-PAGE. Afterwards, the gels were subjected to autoradiography using X-OMAT BT film (Kodak) and then stained with Coomassie Brilliant Blue.

\section{Mass spectrometry}

To identify the autophosphorylation sites of kinases, samples were separated with SDS-PAGE. The bands corresponding to kinases were excised from the gel, reduced with $10 \mathrm{mmol} / \mathrm{L}$ DTT and alkylated with $55 \mathrm{mmol} / \mathrm{L}$ iodoacetamide. The in-gel digestion was carried out by adding the sequence-grade modified trypsin (Promega) in $50 \mathrm{mmol} / \mathrm{L} \mathrm{am}$ monium bicarbonate at $37^{\circ} \mathrm{C}$ overnight. The peptides were extracted twice with $1 \%$ formic acid in $50 \%$ acetonitrile aqueous solution for $30 \mathrm{~min}$. The extraction was then centrifuged in a speedvac to reduce the sample volume. The sample was loaded onto a homemade $\mathrm{C} 18$ column $(100 \mathrm{~mm} \times 100 \mu \mathrm{m})$ packed with Sunchrom packing material (SP-120-3-ODS-A, $3 \mu \mathrm{m}$ ) for the separation of peptide products, and the nano-LC-ESI-MS/MS analysis was carried out subsequently. The peptides were eluted from the HPLC column with a gradient of $0 \%-90 \%$ of Buffer B (acetonitrile: water: acetic acid = 80:19.9:0.1, volume ratio) mixed with Buffer A (acetonitrile: water: acetic acid = 5:94.9:0.1, volume ratio) at a flow rate of approximately $500 \mathrm{~nL} / \mathrm{min}$. The eluted peptides were sprayed directly from the tip of the capillary column to the LTQ mass spectrometer (Thermo Finnigan) for mass spectrometry analysis. The LTQ mass spectrometer was operated in the data-dependent mode in which the initial MS scan recorded the mass to charge $(\mathrm{m} /$ z) ratios of ions over the mass range from $350 \mathrm{Da}$ to $1800 \mathrm{Da}$. The five most abundant ions were automatically selected for subsequent collision-activated dissociation with the multistage activation enabled. All MS/MS data were searched against a database with MsStt7d-KD protein sequence using the SEQUEST (v.28) program (Thermo). The SEQUEST outputs were then analyzed using the commercial software BioWorks (v.3.3.1) (Thermo).

\section{Western blot}

The MsStt7d-KD protein samples before or after phosphorylation reactions were separated by SDS-PAGE and then transferred to the PVDF membranes. The membranes were blocked with nonfat milk (Blotting-Grade Blocker, BIO-RAD) and immunoblotted with anti-phosphothreonine antibody (Cell Signaling Technology), anti-phosphoserine antibody (Millipore) and anti-phosphotyrosine antibody (Cell Signaling 
Technology), respectively. The horseradish peroxidase (HRP)-conjugated secondary antibody and 3, 3'-Diaminobenzidine (DAB) substrate were used for the detection of signals on the blot.

\section{ACCESSION NUMBERS}

Sequence data from this article can be found in the NCBI databank under the following accession numbers: XP_002504185 (MsStt7d), Q84V18 (CrStt7), NP_564946 (AtSTN7), XP_002499717 (MsStt7a), XP_002504036 (MsStt7b), XP_002500340 (MsStt7c), XP_002505498 (MsStt7e), XP_001420129 (OIStt7), BAJ86343 (HvSTN7), NP_001056233 (OsSTN7), NP_001148075 (ZmSTN7), NP_002721 (PKA), NP_057065.2 (CaMK2a), AAA96795 (CKIla), AAP36159 (CDK2), 1IRK_A (Irk) and 1FMK_A (SRC). Coordinates for protein structures can be found in the Protein Data Bank under the following accession numbers: 1QMZ (active CDK2), 1HCL (inactive CDK2), 1IR3 (Irk). Coordinates and structure factors for MsStt7d-KD (PDB: 4IX3), MsStt7d-KD-ADP (PDB: 4IX4), MsStt7d-KD-ATP (PDB: 4IX6), MsStt7d-KD-AMP-PNP (PDB: 4IX5) have been deposited in the Protein Data Bank.

\section{ACKNOWLEDGEMENTS}

We thank the staffs at Shanghai Synchrotron Radiation Facility (SSRF) as well as Yi Han and Shengquan Liu at the X-ray core facility at the Institute of Biophysics (IBP), Chinese Academy of Sciences (CAS) for their technical support during crystal screening and data collection. We also thank Hongmei Zhang for the assistance with molecular biology and biochemistry, Zhensheng Xie and Fuquan Yang at the Proteomic Core Facility Center of IBP, CAS and Jieyuan Liu at the Center of Biomedical Analysis of Tsinghua University for their technical assistance at the Mass Spectrometry analysis. This work was supported by the National Basic Research Program (973 Program) (Nos. 2011CBA00902 and 2011CBA00903) awarded to W. R. C. and Z. F. L., respectively, and the National Natural Science Foundation (Grant No. 31021062).

\section{ABBREVIATIONS}

AMP-PNP, adenosine 5'-( $\beta, \gamma$-imido) triphosphate; AtLhcb1, lightharvesting protein Lhcb1 from Arabidopsis thaliana; AtSTN7, STN7 from Arabidopsis thaliana; CaMK2 $\alpha$, calcium/calmodulin-dependent protein kinase type II subunit $\alpha$ isoform 1 from Homo sapiens; CDK2, cyclin-dependent kinase 2 from Homo sapiens; CKIla, casein kinase II subunit a from Mus musculus; CrStt7, Stt7 from Chlamydomonas reinhardtii; HvSTN7, STN7 from Hordeum vulgare; Irk, Tyr kinase domain of insulin receptor from Homo sapiens; KD, kinase domain; LHCII, major light-harvesting complex II; LTR, long-term response; MsLhcp4, light-harvesting protein Lhcp4 from Micromonas sp. RCC299; MsStt7a-d, Stt7 homolog from Micromonas sp. RCC299; OIStt7, Stt7 from Ostreococcus lucimarinus; OsSTN7, STN7 from Oryza sativa; PKA, cAMP-dependent protein kinase catalytic subunit $\alpha$ isoform 1 from Homo sapiens; PSI, photosystem I; PSII, photosystem II; SRC, Tyr protein kinase C-Src from Homo sapiens; ZmSTN7, STN7 from Zea mays

\section{COMPLIANCE WITH ETHICS GUIDELINES}

Jiangtao Guo, Xuepeng Wei, Mei Li, Xiaowei Pan, Wenrui Chang and Zhenfeng Liu declare that they have no conflict of interest.
This article does not contain any studies with human or animal subjects performed by any of the authors.

\section{REFERENCES}

Adams, P.D., Afonine, P.V., Bunkoczi, G., Chen, V.B., Davis, I.W., Echols, N., Headd, J.J., Hung, L.W., Kapral, G.J., Grosse-Kunstleve, R.W., et al. (2010). PHENIX: a comprehensive Python-based system for macromolecular structure solution. Acta Crystallogr D 66, 213-221.

Allen, J.F. (1992). Protein phosphorylation in regulation of photosynthesis. Biochim Biophys Acta 1098, 275-335.

Allen, J.F., Bennett, J., Steinback, K.E., and Arntzen, C.J. (1981). Chloroplast protein phosphorylation couples plastoquinone redox state to distribution of excitation energy between photosystems. Nature 291, 25-29.

Allen, J.F., and Race, H.L. (2002). Will the real LHC II kinase please step forward? Sci STKE 2002, pe43.

Altschul, S.F., Madden, T.L., Schaffer, A.A., Zhang, J., Zhang, Z., Miller, W., and Lipman, D.J. (1997). Gapped BLAST and PSI-BLAST: a new generation of protein database search programs. Nucleic Acids Res 25, 3389-3402.

Battye, T.G., Kontogiannis, L., Johnson, O., Powell, H.R., and Leslie, A.G. (2011). iMOSFLM: A new graphical interface for diffractionimage processing with MOSFLM. Acta Crystallogr D 67, 271-281.

Bellafiore, S., Barneche, F., Peltier, G., and Rochaix, J.D. (2005). State transitions and light adaptation require chloroplast thylakoid protein kinase STN7. Nature 433, 892-895.

Bennett, J. (1991). Protein phosphorylation in green plant chloroplasts. Annu Rev Plant Physiol Plant Mol Biol 42, 281-311.

Bonardi, V., Pesaresi, P., Becker, T., Schleiff, E., Wagner, R., Pfannschmidt, T., Jahns, P., and Leister, D. (2005). Photosystem II core phosphorylation and photosynthetic acclimation require two different protein kinases. Nature 437, 1179-1182.

Bonaventura, C., and Myers, J. (1969). Fluorescence and oxygen evolution from Chlorella pyrenoidosa. Biochim Biophys Acta 189, 366-383.

Bossemeyer, D. (1994). The glycine-rich sequence of protein kinases: a multifunctional element. Trends Biochem Sci 19, 201-205.

Brown, N.R., Noble, M.E., Endicott, J.A., and Johnson, L.N. (1999). The structural basis for specificity of substrate and recruitment peptides for cyclin-dependent kinases. Nat Cell Biol 1, 438-443.

Brunger, A.T., Adams, P.D., Clore, G.M., DeLano, W.L., Gros, P., Grosse-Kunstleve, R.W., Jiang, J.S., Kuszewski, J., Nilges, M., Pannu, N.S., et al. (1998). Crystallography and NMR system: a new software suite for macromolecular structure determination. Acta Crystallogr D 54, 905-921.

CCP4 (1994). The CCP4 suite: programs for protein crystallography. Acta Crystallogr D 50, 760-763.

Coughlan, S., and Hind, G. (1987). Phosphorylation of thylakoid proteins by a purified kinase. J Biol Chem 262, 8402-8408.

De Bondt, H.L., Rosenblatt, J., Jancarik, J., Jones, H.D., Morgan, D.O., and Kim, S.H. (1993). Crystal structure of cyclin-dependent kinase 2. Nature 363, 595-602.

DeLano, W.L. (2002). The PyMOL Molecular Graphics System. (San Carlos, CA: DeLano Scientific). http://www.pymol.org

Depège, N., Bellafiore, S., and Rochaix, J.D. (2003). Role of chloro- 
plast protein kinase Stt7 in LHCll phosphorylation and state transition in Chlamydomonas. Science 299, 1572-1575.

Dietzel, L., Brautigam, K., and Pfannschmidt, T. (2008). Photosynthetic acclimation: state transitions and adjustment of photosystem stoichiometryfunctional relationships between short-term and long-term light quality acclimation in plants. FEBS J 275, 1080-1088.

Emsley, P., and Cowtan, K. (2004). Coot: model-building tools for molecular graphics. Acta Crystallogr D 58, 1948-1954.

Endicott, J.A., Noble, M.E., and Johnson, L.N. (2012). The structural basis for control of eukaryotic protein kinases. Annu Rev Biochem 81, 587-613.

Evans, P. (2006). Scaling and assessment of data quality. Acta Crystallogr D 62, 72-82.

Hanks, S.K., and Hunter, T. (1995). Protein kinases 6. The eukaryotic protein kinase superfamily: kinase (catalytic) domain structure and classification. FASEB J 9, 576-596.

Hubbard, S.R. (1997). Crystal structure of the activated insulin receptor tyrosine kinase in complex with peptide substrate and ATP analog. EMBO J 16, 5572-5581.

Jeffrey, P.D., Russo, A.A., Polyak, K., Gibbs, E., Hurwitz, J., Massague, J., and Pavletich, N.P. (1995). Mechanism of CDK activation revealed by the structure of a cyclinA-CDK2 complex. Nature 376 , 313-320.

Kargul, J., and Barber, J. (2008). Photosynthetic acclimation: structural reorganisation of light harvesting antenna--role of redox-dependent phosphorylation of major and minor chlorophyll a/b binding proteins. FEBS J 275, 1056-1068.

Laskowski, R.A., MacArthur, M.W., Moss, D.S., and Thornton, J.M. (1993). PROCHECK: a program to check the stereochemical quality of protein structures. J Appl Cryst 26, 283-291.

Lemeille, S., Willig, A., Depege-Fargeix, N., Delessert, C., Bassi, R., and Rochaix, J.D. (2009). Analysis of the chloroplast protein kinase Stt7 during state transitions. PLoS Biol 7, e45.

Lin, Z.F., Lucero, H.A., and Racker, E. (1982). Protein kinases from spinach chloroplasts. I. Purification and identification of two distinct protein kinases. J Biol Chem 257, 12153-12156.

Lindberg, R.A., Quinn, A.M., and Hunter, T. (1992). Dual-specificity protein kinases: will any hydroxyl do? Trends Biochem Sci 17, 114-119.

Madhusudan, Trafny, E.A., Xuong, N.H., Adams, J.A., Ten Eyck, L.F., Taylor, S.S., and Sowadski, J.M. (1994). cAMP-dependent protein kinase: crystallographic insights into substrate recognition and phosphotransfer. Protein Sci 3, 176-187.

McNicholas, S., Potterton, E., Wilson, K.S., and Noble, M.E.M. (2011). Presenting your structures: the CCP4mg molecular-graphics software. Acta Crystallogr D 67, 386-394.

Mendelow, M., Prorok, M., Salerno, A., and Lawrence, D.S. (1993). ATPase-promoting Dead End Inhibitors of the CAMP-dependent Protein Kinase. J Biol Chem 268, 12289-12296.

Murata, N. (1969). Control of excitation transfer in photosynthesis. I. Light-induced change of chlorophyll a fluorescence in Porphyridium cruentum. Biochim Biophys Acta 172, 242-251.

Murshudov, G.N., Vagin, A.A., and Dodson, E.J. (1997). Refinement of Macromolecular structures by maximum-likelihood method. Acta Crystallogr D 53, 240-255.

Nolen, B., Taylor, S.S., and Ghosh, G. (2004). Regulation of Protein Kinases Controlling Activity through Activation Segment Conforma- tion. Mol Cell 15, 661-675.

Oh, M.H., Wang, X., Clouse, S.D., and Huber, S.C. (2012). Deactivation of the Arabidopsis BRASSINOSTEROID INSENSITIVE 1 (BRI1) receptor kinase by autophosphorylation within the glycinerich loop. Proc Natl Acad Sci U S A 109, 327-332.

Otwinowski, Z., and Minor, W. (1997). Processing of X-ray diffraction data collected in oscillation mode. Methods Enzymol 276, 307-326.

Pesaresi, P., Hertle, A., Pribil, M., Kleine, T., Wagner, R., Strissel, H., Ihnatowicz, A., Bonardi, V., Scharfenberg, M., Schneider, A., et al. (2009). Arabidopsis STN7 kinase provides a link between short- and long-term photosynthetic acclimation. Plant Cell 21, 2402-2423.

Reiland, S., Messerli, G., Baerenfaller, K., Gerrits, B., Endler, A., Grossmann, J., Gruissem, W., and Baginsky, S. (2009). Largescale Arabidopsis phosphoproteome profiling reveals novel chloroplast kinase substrates and phosphorylation networks. Plant Physiol 150, 889-903.

Rochaix, J.D. (2007). Role of thylakoid protein kinases in photosynthetic acclimation. FEBS Lett 581, 2768-2775.

Schulze-Gahmen, U., De Bondt, H.L., and Kim, S.H. (1996). Highresolution crystal structures of human cyclin-dependent kinase 2 with and without ATP: bound waters and natural ligand as guides for inhibitor design. J Med Chem 39, 4540-4546.

Snyders, S., and Kohorn, B.D. (1999). TAKs, thylakoid membrane protein kinases associated with energy transduction. J Biol Chem 274, 9137-9140.

Snyders, S., and Kohorn, B.D. (2001). Disruption of thylakoid-associated kinase 1 leads to alteration of light harvesting in Arabidopsis. J Biol Chem 276, 32169-32176.

Vainonen, J.P., Hansson, M., and Vener, A.V. (2005). STN8 protein kinase in Arabidopsis thaliana is specific in phosphorylation of photosystem II core proteins. J Biol Chem 280, 33679-33686.

Vener, A.V., van Kan, P.J.M., Rich, P.R., Ohad, I., and Andersson, B. (1997). Plastoquinol at the quinol oxidation site of reduced cytochrome bf mediates signal transduction between light and protein phosphorylation: Thylakoid protein kinase deactivation by a singleturnover flash. Proc Natl Acad Sci U S A 94, 1585-1590.

Wagner, R., Dietzel, L., Brautigam, K., Fischer, W., and Pfannschmidt, T. (2008). The long-term response to fluctuating light quality is an important and distinct light acclimation mechanism that supports survival of Arabidopsis thaliana under low light conditions. Planta 228, 573-587.

Ward, N.E., and O'Brian, C.A. (1992). The intrinsic ATPase activity of protein kinase $C$ is catalyzed at the active site of the enzyme. Biochemistry 31, 5905-5911.

Welburn, J.P.I., Tucker, J.A., Johnson, T., Lindert, L., Morgan, M., Willis, A., Noble, M.E.M., and Endicott, J.A. (2006). How tyrosine 15 phosphorylation inhibits the activity of cyclin-dependent kinase 2-cyclin A. J Biol Chem 282, 3173-3181.

Willig, A., Shapiguzov, A., Goldschmidt-Clermont, M., and Rochaix, J.D. (2011). The phosphorylation status of the chloroplast protein kinase STN7 of Arabidopsis affects its turnover. Plant Physiol 157, 2102-2107.

Wollman, F.A. (2001). State transitions reveal the dynamics and flexibility of the photosynthetic apparatus. EMBO J 20, 3623-3630.

Zito, F., Finazzi, G., Delosme, R., Nitschke, W., Picot, D., and Wollman, F.A. (1999). The Qo site of cytochrome b6f complexes controls the activation of the LHCII kinase. EMBO J 18, 2961-2969. 